\title{
A Simple Mathematical Model for Information Rate of Active Transport Molecular Communication
}

\author{
Nariman Farsad, Andrew W. Eckford \\ Department of Computer Science and Engineering \\ York University \\ 4700 Keele Street, Toronto, ON, Canada M3J 1P3 \\ Email: nariman@cse.yorku.ca, aeckford@yorku.ca
}

\author{
Satoshi Hiyama and Yuki Moritani \\ Research Laboratories \\ NTT DOCOMO Inc. \\ Yokosuka, Kanagawa, Japan \\ Email: \{hiyama, moritani\}@nttdocomo.co.jp
}

\begin{abstract}
In molecular communication, gaps in the underlying theoretical and mathematical framework create numerous challenges. Currently, most researchers rely on simulations to study these systems. However, simulations can be time consuming and impractical. Moreover, due to the complexity and dependencies present in these systems, deriving a mathematical framework that can capture the essence of molecular communication systems is also challenging. In this work, we derive a simple mathematical model, based on some independence assumptions, to estimate the information rate of a molecular communication system employing active transport propagation. We show that the presented model estimates the simulated information rate closely for small communication time intervals. We also use the derived mathematical model to design and verify an optimal loading area that would maximize the information rate.
\end{abstract}

\section{INTRODUCTION}

Molecular communication [1] is a new and emerging field of science where molecules are used to transfer information over very small distances, typically on the order of microor nanometers. Molecular communication systems are complex, and traditional communication analysis techniques can not be applied to them directly. Furthermore, due to gaps in their underlying theoretical and mathematical framework, researchers sometimes rely on computer simulations to study these communication systems. However, running simulations can be time consuming and impractical. Therefore, it is desirable to find a mathematical framework for studying these systems. Moreover, deriving a mathematical model, specially for molecular communication in confined space, is extremely difficult because of the complexity and dependencies present in these systems. In this work, with the help of some independence assumptions, we first present a simple mathematical representation of molecular communication systems in confined space, that can be employed to estimate the information rate of active transport propagation. We then use the proposed model to design a loading area or transmission zone that would maximize the information transfer rate.

In molecular communication there are two major propagation schemes: passive transport and active transport. In passive transport, the information carrying particles diffuse in the confined microfluidic channel and follow a Brownian motion from the transmission zone to the receiver zone. In active transport, the information particles are transported actively using molecular motors such as kinesin. For example in [2], microtubule filaments moving over a glass substrate covered with stationary kinesin motors is proposed as an active transport scheme for molecular communication systems. In this work we consider active transport propagation scheme based on [2] to derive our mathematical model.

Notable works employing simulation or mathematical models to study molecular communication systems include a general formulation of molecular communication as a timing channel under Brownian motion [3], [4], an analysis of information transfer rates using molecular motors [5], [6], mathematical channel models for continuous diffusion [7], and a simple model comparing the achievable information rates of passive transport using Brownian motion to that of active transport using microtubule filaments moving over a molecular motor track [8], [9].

While in [8], [9] we rely on simulations to obtain the information rate, in this paper we focus on deriving a mathematical model which could eliminate the need for simulations. As the main contribution of this paper, we present a simple mathematical model based on the active transport propagation system presented in [9]. For small values of time duration per communication session (upto 1000s), we conclude that our mathematical model can closely estimate the information rate. For larger time durations (above 1000s) the accuracy of the estimation drops as the time duration increases. This error in estimation is because of the simplifying independence assumptions in the proposed mathematical model. As time per communication session increases, these assumptions become less realistic and result in larger errors in estimation. As an application of this contribution, we use this model to find and verify an optimal design of the loading area; this optimization leads to large improvements in performance, and would be computationally intensive to find without our model.

The rest of this paper is summarized as follows. In Section II-A we first present a brief overview of molecular communication environment. We then discuss the encoding scheme, information rate and channel capacity in Section II-B. Our proposed mathematical model for estimation of information rate is proposed in Section II-C. In Section II-D we optimize the transmission area using the proposed mathematical model. We verify our mathematical model by comparing the results 


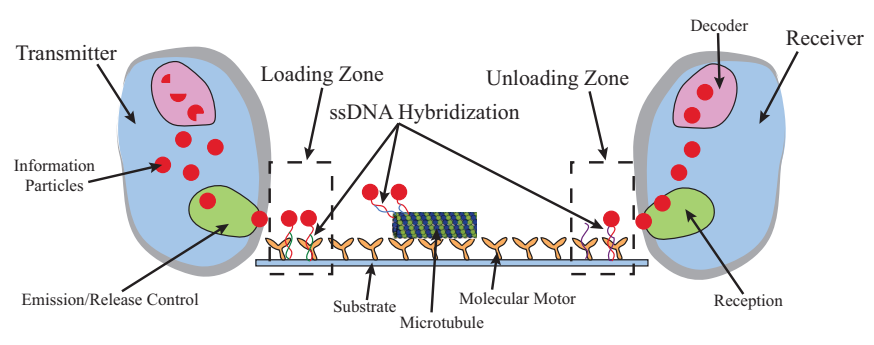

Fig. 1. Depiction of the communication environment.

against software simulation in Section III. Finally, we present our concluding remarks in Section IV.

\section{COMmunication ENVIRONMENT AND Mathematical Model}

\section{A. Molecular Communication Environment}

Our molecular communication environment is similar to the ones in [8], [9], [2]. We use a rectangular propagation environment (with slightly rounded corners to assist the simulation), consisting of a transmission or loading zone and a receiver or unloading zone. Message-bearing particles originate at the loading zone, and propagate until they arrive at the unloading zone. These particles are initially assumed to be anchored to the loading zone until microtubule filaments, moving over molecular motor tracks that cover the whole environment, pick up and transport the information carrying particles from the loading zone to the unloading zone.

As in [2], we assume that the microchannel environment is lined with static kinesin molecular motors, and that these motors cause microtubule filaments to propagate along their surface. The motion of the microtubule is largely regular (compared to pure Brownian Motion), although the effects of Brownian motion cause random fluctuations. The loading and unloading mechanics are assumed to be the same as those proposed in [2]. The particles are anchored to the loading zone through a single stranded DNA (ssDNA) hybridization bonds, and do not move until they are picked up by a microtubule filament. The pick-up and drop-off mechanisms are also assumed to be carried out through ssDNA hybridization bonds. The starting location of the microtubule is assumed to be random and uniformly distributed across the entire propagation area. Moreover, the initial directional angle for the motion of the microtubule is selected uniformly at random from the range $[0,2 \pi]$, and microtubules are assumed to be initially unloaded. This process is summarized in Figure 1 and the reader is referred to [2] for detailed explanation.

In order to capture the loading process, in the simulation and the mathematical model, we use the grid loading structure proposed in [9]. For loading an information particle, the microtubule filament must drive close to the anchored particle. Therefore, we divide the loading zone into a square grid, where the length of each square in the grid is the same as the diameter of the particles. We then distribute particles randomly and uniformly between the squares in the grid. If a microtubule

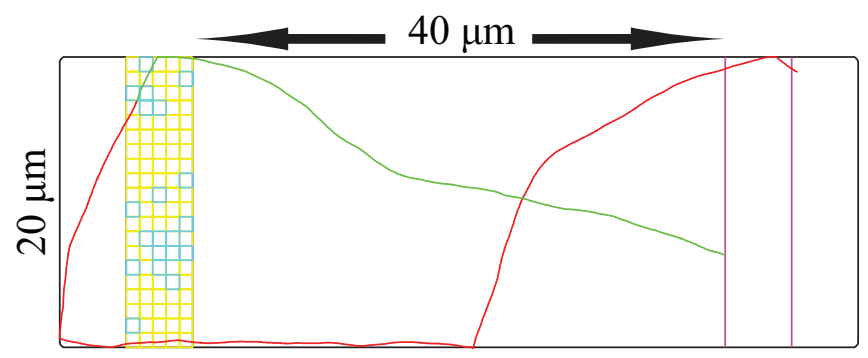

$60 \mu \mathrm{m}$

Fig. 2. A sample trajectory of active transport. The path of the microtubule is the line in the middle of the rectangular channel. The red portion corresponds to the path of the unloaded microtubule, while the green portion corresponds to the path of the loaded microtubule. The grid loading structure is on the left hand side and the unloading strip is on the right hand side. The cyan squares in the loading zone represent areas that contain an anchored particle which yellow squares represent areas with no particles.

enters a square which is occupied by a particle, we assume the microtubule loads that particle given it has an empty loading slot available. In general, based on laboratory experiments we assume that the microtubules can load multiple particles and the maximum number of particles a microtubule can load is given by half of its length divided by the diameter of the particles. For unloading, we assume all the loaded particles are unloaded as soon as a microtubule enters the unloading zone. Figure 2 shows a sample trajectory with the loading and unloading mechanism. Notice that the loading and the unloading zone in this figure are rectangular strips across the width of the channel. We refer to this particular shape of the loading and the unloading zone as the strip loading zone and the strip unloading zone, respectively.

\section{B. Achievable Information Rate in Molecular Communication}

In this section we describe the channel capacity and achievable information rates, which are an important part of any communication system, and discuss how they can be applied to molecular communication channels. Previous work has considered molecular communication either as a timing channel problem (i.e., where information is encoded in the times when molecules are released) [3], [6], or as an inscribed matter problem (i.e., where information is encoded by transmitting custom-made particles, such as specific strands of DNA). However, in this paper, we consider information transmission as a mass transfer problem - in other words, a message is transmitted by moving a number of particles from the transmitter to the receiver.

In the simplest possible conception of this scheme, the particles themselves are not information-bearing, and a message is conveyed in the number of particles released by the transmitter. For example, if a maximum of three particles may be used, from a traditional communication system perspective, we may form messages two bits long (i.e., $\log _{2} 4$ ): " 00 " for 0 particle, " 01 " for 1 particle, " 10 " for 2 particles, and "11" for 3 particles. However, this message might not be perfectly conveyed to the receiver: given a time limit $T$ for the 


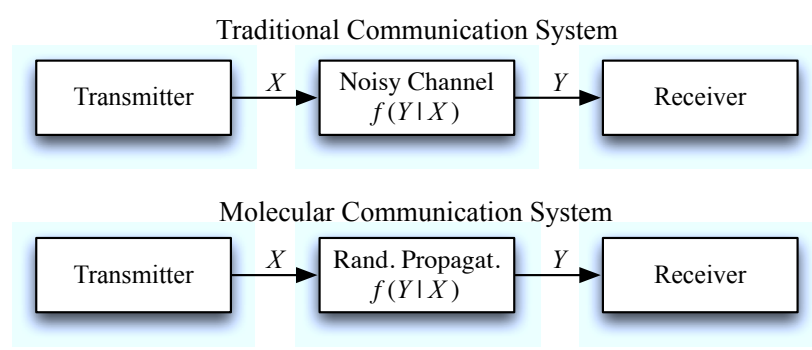

Fig. 3. Traditional and molecular communication channels. In traditional communication the noisy channel limits the channel capacity, while in molecular communication the random propagation of information particles limits the channel capacity.

communication session, it is possible that some of the particles will not arrive at the receiver after $T$ has elapsed.

The maximum rate any communication system can reliably transmit information over a noisy channel is bounded by a limit called channel capacity [10]. In traditional communication systems, a source (transmitter) has a set of possible transmission symbols $\mathcal{X}$ and transmits a symbol $X \in \mathcal{X}$ per channel use, where, from the channel's perspective, $X$ is a random variable given by probability distribution function (PDF) $f_{X}(x)$. Because of the noise present in the channel, the destination (receiver) might not receive the symbol $X$ correctly. Let $\mathcal{Y}$ be the set of possible message symbols the destination can receive. Note that $\mathcal{X} \subseteq \mathcal{Y}$ since the received symbol might be corrupted such that it is not in $\mathcal{X}$. Therefore, the destination receives a symbol $Y \in \mathcal{Y}$ per channel use, where $Y$ is a random variable given by $\operatorname{PDF} f_{Y}(y)$. This process is summarized in the top portion of Figure 3.

The channel capacity of such communication channel can be calculated using mutual information $I(X ; Y)$, given by

$$
I(X ; Y)=E\left[\log _{2} \frac{f_{Y \mid X}(y \mid x)}{\sum_{x} f_{Y \mid X}(y \mid x) f_{X}(x)}\right],
$$

where, in this example, $f_{Y \mid X}(y \mid x)$ represents the probability of receiving symbol $y$ at the destination, given that symbol $x$ was transmitted by the source; $f_{X}(x)$ represents the probability of transmitting symbol $x$ at the source; and $E[\cdot]$ represents expectation. The channel capacity can be calculated using mutual information as,

$$
C=\max _{f_{X}(x)} I(X ; Y)
$$

where $C$ is the channel capacity, which is represented by the maximum value of mutual information over all possible transmission symbols' probability distributions.

The same concept can be applied to molecular communication systems, as shown in the bottom of Figure 3. Since we have assumed that messages are encoded in the number of particles, let $X$ represent the number of particles released into the medium by the transmitter (i.e. the source symbols), $Y$ represent the number that arrive at the destination (i.e. the symbol at the receiver) once $T$ seconds have elapsed, and $x_{\max }$ be the maximum number of particles the transmitter can release per channel use. In other words, the set of possible transmission symbols are $\mathcal{X}=\left\{0,1,2, \cdots, x_{\max }\right\}$. Just like the traditional communication channel, from the channel's perspective, $X \in \mathcal{X}$ is a discrete random variable given by probability mass function (PMF) $f_{X}(x), Y \in \mathcal{X}$ is also a discrete random variable given by PMF $f_{Y}(y)$, and a channel use is defined as $T$ second intervals between the transmission releases. Therefore in traditional communication system, the received symbols at the receiver are corrupted with noise from the environment, while in the molecular communication system, the received symbols are corrupted because of the random propagation of particles.

Clearly, there exists some PMF $f_{Y \mid X}(y \mid x)$ of the number of arrived particles given the number of transmitted particles. If this PMF is known, we can calculate mutual information for any $f_{X}(x)$. However, in order to calculate the channel capacity we need to find the PMF $f_{X}(x)$ that maximizes mutual information. We can use the Blahut-Arimoto algorithm [11], [12] to find the PMF $f_{X}(x)$ such that, given $f_{Y \mid X}(y \mid x)$, mutual information is maximized. Therefore, if PMF $f_{Y \mid X}(y \mid x)$ is known, we can calculate the channel capacity of the molecular communication system in a straight forward manner.

Finding the PMF $f_{Y \mid X}(y \mid x)$ is non-trivial because of the random motion of particles, as well as the shape and the geometry of the confined molecular communication channel. This PMF can be estimated using Monte Carlo simulations as proposed in [8], [9]. However, these simulations can be time-consuming since for each value of $X \in \mathcal{X}$ and value of $T$, a set of Monte Carlo simulations is necessary to estimate PMF $f_{Y \mid X}(y \mid x)$. In the next section we present a mathematical model that could be used to estimate PMF $f_{Y \mid X}(y \mid x)$ using a simpler set of simulations based on different values of $T$.

\section{Mathematical Modelling}

Assume that our grid loading zone contains $n$ squares (i.e. the maximum number of particles that can be anchored to the loading zone is $n$ ). As explained before, Let $X \leq n$ be the number of particles at the transmission zone in the beginning, and let $Y \leq X$ be the number of particles delivered to the receiver zone after time duration $T$. Let $X_{i}$ be a Bernoulli random variable representing the event where a particle is placed in the $i$ th square for $i=1,2, \cdots, n$. Therefore, if we assume that $X_{i}$ are independent of each other, the probability that an information particle is placed in the $i$ th square is given by

$$
p\left(X_{i}=1\right)=\frac{X}{N}
$$

where particles are distributed uniformly among squares. Note that the independence assumption here is an approximation because it does not satisfy the constraint

$$
X=\sum_{i=1}^{n} X_{i},
$$

Let $V_{i}$ be a Bernoulli random variable representing the event that the $i$ th square is visited by the microtubule in a single trip 


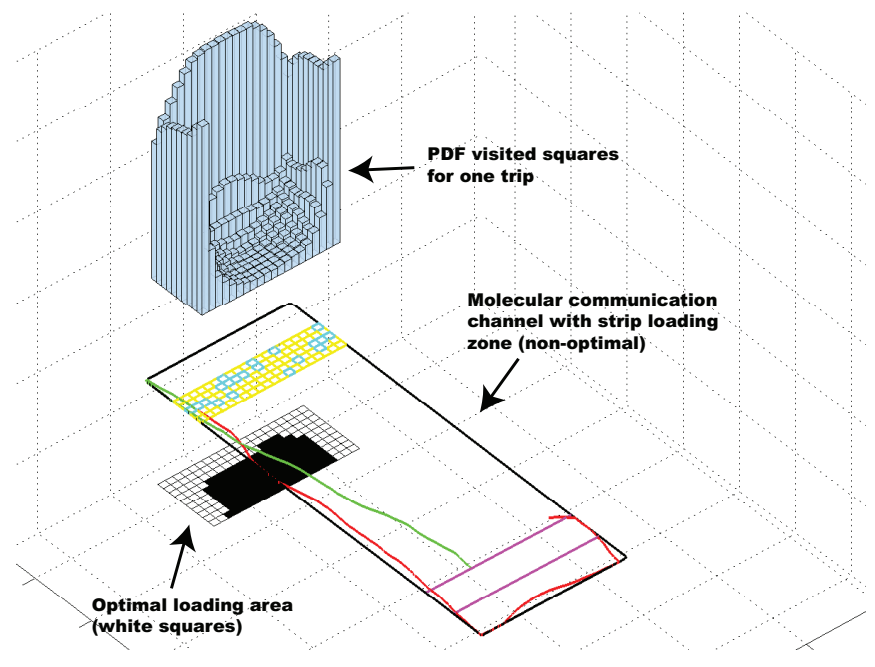

Fig. 4. (Top): Probability distribution of $p\left(V_{i}=1\right)$ for squares of size $1 \mu \mathrm{m}$ to the left side of the loading area. (Middle): Strip loading area (yellow and cyan squares) for $n=100$ squares. (Bottom): Projection of the probability distribution $p\left(V_{i}=1\right)$ on top. The top 100 values of $p\left(V_{i}=1\right)$ are shown in as white squares and they represent the optimal loading area.

from the receiver zone, to the transmission zone, and back. Therefore, $p\left(V_{i}=1\right)$ represents the probability that the $i$ th square is visited and $p\left(V_{i}=0\right)$ the probability that it is not visited. This probability distribution can quickly be calculated using simple simulations for any molecular communication channel. For example, the top part of the Figure 4 shows this probability distribution for squares of size $1 \mu \mathrm{m}$ covering the left side of the microchannel. From the probability distribution, it can be seen that the squares close to the walls are visited the most, which is a property of the motion of the microtubules.

Let $K$ be another random variable representing the number of microtubule trips between the transmission and the receiver zone in time duration $T$. The probability distribution for $K$ is given in [8], and can be quickly calculated for any molecular communication channel using simple simulations. Let $V_{i}^{(k)}$ be a Bernoulli random variable representing the event that the $i$ th square is visited at least once by the microtubule during $k$ trips. Therefore,

$$
p\left(V_{i}^{(k)}=1\right)=1-\left(1-p\left(V_{i}=1\right)\right)^{k},
$$

represents the corresponding probability distribution.

Let $D_{i}^{k}$ be a Bernoulli random variable representing the event that a particle from the $i$ th square is delivered to the destination after $k$ trips. Then, the probability distribution of $D_{i}^{k}$ is given by

$$
p\left(D_{i}^{(k)}=1\right)=p\left(V_{i}^{(k)}=1\right) p\left(X_{i}=1\right),
$$

assuming $p\left(V_{i}^{(k)}=1\right)$ and $p\left(X_{i}=1\right)$ are independent. This independence assumption is not accurate since $p\left(X_{i}=1\right)$ changes depending on the number of particles already delivered in previous trips. In general this assumption becomes less accurate as the number of trips $k$ increases or in other words the channel time duration $T$ increases. Let $Y^{(k)}$ be the total number of particles delivered to the unloading zone during $k$ tips. Then, $Y^{(k)}$ is given by

$$
Y^{(k)}=\min \left(\sum_{i=1}^{n} D_{i}^{k}, X\right),
$$

for any given $X$. Since $\sum_{i=1}^{n} D_{i}^{k}$ represents a PoissonBinomial distribution, its corresponding probability distribution can be calculated using [13]. Finally, we can calculate $\operatorname{PMF} f_{Y \mid X}(Y \mid X)$ as

$$
f_{Y \mid X}(Y \mid X)=\sum_{k \in K} p\left(Y^{(k)} \mid X\right) p(k),
$$

where $p\left(Y^{(k)} \mid X\right)$ is the probability mass function of $Y^{(k)}$ given in Equation (7), and $p(k)$ is the probability mass function of $K$, the number of trips between the transmitter and receiver during the time duration $T$.

The benefits of this model is twofold. First, it can be employed to quickly estimate the information rate of any molecular communication system, and although it relies on simulations for calculating the probability distributions of $V_{i}$ and $K$, these simulations are very simple and can be performed on an average computer quickly. Second, because of the model's simplicity, various design problems can be solved, for example, in the next section we use this model to generate an optimal transmission zone. The only drawback of this model is that the resulting $\mathrm{PMF}, f_{Y \mid X}(Y \mid X)$, is not as accurate as the one derived using Monte Carlo simulation scheme used in [6], [9].

\section{Improving Information rate}

We can improve channel capacity if we transfer information particles from the transmitter to the receiver quicker on average. We use our mathematical model presented in previous section, and the fact that the microtubules mostly move along the walls of the molecular communication channel, to optimize the loading area. Recall from top part of the Figure 4 that microtubules mostly move along the walls, and therefore $p\left(V_{i}=1\right)$ (probability that a square is visited in one microtubule trip) is higher for squares close to the walls of the molecular communication channel. An information particle is picked up from the transmission zone, and delivered to the receiver zone, if the corresponding square is visited. Therefore, we want to find squares with maximum $p\left(V_{i}=1\right)$, which are squares that have the highest probability of being visited during one trip. In Figure 4, we plot the probability distribution of all the squares of length $1 \mu \mathrm{m}$ to the left of transmission area (the bar plot on the top). The middle plot in Figure 4 shows our original strip loading area with 100 squares presented in Figure 2. Notice that the squares in the middle of the strip loading zone have low probability of being visited in a single microtubule trip. The bottom plot shows the projection of the probability distribution function of $p\left(V_{i}=1\right)$. The first 100 squares with highest probabilities are shown as white squares, and the rest of the squares are shown in black. Note that in this projection, the minimum distance between the transmission 


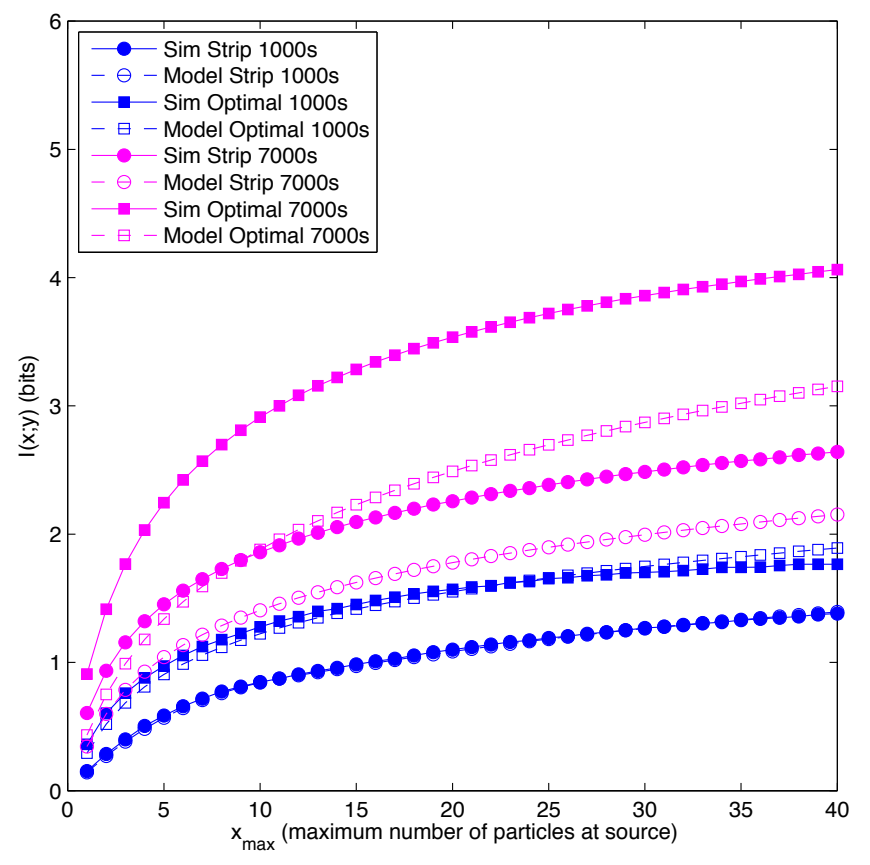

Fig. 5. Mutual information (i.e. information rate) plot. Strip loading zone plots are presented as circles and optimal loading zone plots are presented as squares. The solid markers indicate simulated information rate and the empty markers present the information rate calculated using our model. The blue plots present the communication time duration of 1000 s and the magenta plots represent a time duration of 7000 s. Note the very high accuracy of the model at $1000 \mathrm{~s}$

area and the receiver area is still $40 \mu \mathrm{m}$ and we have not moved the transmission and receiver physically closer. Finally, according to our mathematical model, this white area is the optimal loading zone that will give us the highest information rate because probability of visiting and picking up particles is highest.

\section{RESULTS}

In this section, we compare the information rates obtained through simulation to the information rates obtained using our mathematical model. In order to make this comparison, we consider a rectangular propagation environment, with the dimensions $20 \mu \mathrm{m}$ by $60 \mu \mathrm{m}$ presented in Figure 2 . The transmission area is the strip on the left while the receiver area is on the strip right and the separation between the transmission zone and the receiver zone is $40 \mu \mathrm{m}$. We assume the diameter of the information particles is $1 \mu \mathrm{m}$ and there are 100 squares in the grid loading area (i.e. there could be as much as 100 squares in the loading strip). We also use our mathematical model to generate an optimal loading area that would maximize the information rate as explained in the previous section. This optimal loading area is represented by the white squares in the bottom of the Figure 4.

For Monte Carlo simulation of the motion of the microtubules, we use the same technique proposed in [9] with the following parameters: simulation time steps of $\Delta T=0.1$ seconds, microtubule diffusion coefficient $D=2.0 \cdot 10^{-3}$ $\mu \mathrm{m}^{2} / \mathrm{s}$, average speed of the microtubule $v_{\text {avg }}=0.5 \mu \mathrm{m} / \mathrm{s}$, and persistence length of the microtubules trajectory $L_{p}=111$ $\mu \mathrm{m}$. We also assume the size of the information particles is $1 \mu \mathrm{m}$, the average length of the microtubules is $10 \mu \mathrm{m}$, and each microtubule can load up to 5 information particles in one trip from the transmission zone to the receiver zone. These parameters are all selected based on experimental observations of ssDNA covered microtubules moving over a kinesin covered substrate.

In our simulations we assume the set of possible transmission symbols are $\mathcal{X}=\left\{0,1,2, \cdots, x_{\max }\right\}$, for some value of $x_{\max }$, where a transmission symbol $X \in \mathcal{X}$ is represented by release of $X$ information particles into the medium. In the case of active transport, all the released particles will be randomly distributed and stationary at the transmission zone until they are picked up for delivery by a microtubule. By simulating the motion of the microtubules many times, we derive the PMF $f_{Y \mid X}(y \mid x)$ for both passive and active transport. We then use Blahut-Arimoto algorithm [11], [12] to find the PMF $f_{X}(x)$, that would maximize the mutual information, and hence calculate the channel capacity for each propagation scheme. We repeat the same process using our mathematical model to estimate PMF $f_{Y \mid X}(y \mid x)$ and then compare the results.

Figure 5 shows the mutual information (i.e. maximum achievable information rate) of two different loading zones: strip loading zone, shown using circles, and the optimal loading zone, shown using squares. The mutual information is plotted against the $x_{\max }$ which represents the maximum number of possible transmission symbols. The mutual information is calculated by using both simulation (solid markers) and the mathematical model (empty markers). As we can see for small time durations, the mathematical model estimates the simulated mutual information closely. However, as time duration increases, the difference between the mutual information obtained through simulations and the mathematical model increases. This error is a by product of the independence assumptions made during the derivation of our mathematical model. Finally, we can see that the optimal loading zone achieves a much higher information rate compared to the strip loading zone as proposed by our analysis of our mathematical model.

TABLE I

Number OF SIMULATIONS REQUIRED TO ESTIMATE $f_{Y \mid X}(y \mid x)$

\begin{tabular}{cc}
\hline \hline Method of PMF Estimation & Number of Computer Simulations \\
\hline Monte Carlo Simulation & $2 \times 2 \times 40=160$ \\
Mathematical Model & 3 \\
\hline
\end{tabular}

Finally, we compare the number of computer simulations required to estimate PMF $f_{Y \mid X}(y \mid x)$, for the plots in Figure 5, using both Monte Carlo simulations and the proposed mathematical model. The results are summarized in Table I. Since there are 40 values of $x_{\max }$ and two time durations of 1000s and 7000s, when Monte Carlo simulation is used to estimate the PMF $f_{Y \mid X}(y \mid x)$, there are 80 sets of simulations 
required to generate the plot for the strip loading area and another 80 sets of simulations to generate the results for optimal loading area. However, using our mathematical model, two sets of simulation are necessary to calculate the PMF for $K$, the number of microtubule trips from the loading zone to the unloading zone in times 1000s and 7000s, and one set of simulations to calculate the PMF for $V_{i}$, the probability that the $i$ th grid square is visited in one trip. These simulations can be used for both strip and optimal loading area and as the result no extra simulations are necessary.

Furthermore, when Monte Carlo simulation is employed, as the number of information particles released by the transmitter increases, the actual simulation times increases. For example, simulating one particle at the source take much less time than simulating 40 particles at the source. In this case, when one particle is assumed to be released, the simulation runs until time duration $T$ is simulated or until the single particle is delivered, while it must run until time duration $T$ is simulated or 40 particle are delivered when 40 particles are assumed to be released. However, using the proposed mathematical model, the simulation times are constant regardless of the number of particles released by the transmitter. Therefore, using the proposed mathematical model the channel capacity can be calculated much quicker than the Monte Carlo simulations proposed in [6], [9]. For example, using simulations written in Matlab [14], and average desktop computers equipped with Intel Core Due processors with different speeds ranging from $2.5 \mathrm{GHz}$ to $3.0 \mathrm{GHz}$, we used 40 different CPU cores to generate the Monte Carlo simulation results shown in Figure 5 , for both the strip and optimal loading area, in about two weeks (almost one week for each loading zone). However, the plots based on the mathematical model were generated using three CPU cores in about a day for both the strip and the optimal loading area.

\section{CONCLUSION}

In this work we considered a molecular communication system, in a rectangular confined space, employing active transport as proposed in [2]. In order to estimate the channel capacity of such a communication systems, previous works [6], [9], had relied on Monte Carlo simulations of the motion of microtubule filaments. These simulations can be time consuming since for each set of possible transmission symbols as well as channel time durations, a different set of simulation is necessary. We proposed a mathematical model that could be used to estimate the channel capacity of these systems. The benefits of the proposed model are three folds. First, using the mathematical model a fewer number of simulations is required to estimate the channel capacity. Second, using the insights provided by the model an optimal loading area is derived that increases the channel capacity of the molecular communication channel. The channel capacity estimation obtained using the proposed mathematical model is very close to the Monte Carlo simulation estimated channel capacity for small channel time durations. However, as channel time duration increases the error in the proposed mathematical model increases. Finally, the proposed mathematical model could be expanded to any other molecular communication channel quickly and could possibly be used to optimize the shape of the microchannel itself.

\section{REFERENCES}

[1] S. Hiyama and Y. Moritani, "Molecular communication: harnessing biochemical materials to engineer biomimetic communication systems," Nano Communication Networks, vol. 1, no. 1, pp. 20-30, 2010.

[2] S. Hiyama, R. Gojo, T. Shima, S. Takeuchi, and K. Sutoh, "Biomolecular-motor-based nano- or microscale particle translocations on dna microarrays," Nano Letters, vol. 9, no. 6, pp. 2407-2413, 2009.

[3] A. W. Eckford, "Nanoscale communication with brownian motion," in Proc. of the 41st Annual Conference on Information Sciences and Systems, (Baltimore, MD), pp. 160-165, 2007.

[4] B. Atakan and O. Akan, "An information theoretical approach for molecular communication," in Proc. of the 2nd International Conference on Bio-Inspired Models of Network, Information and Computing Systems, (Budapest, Hungary), pp. 33-40, 2007.

[5] M. J. Moore, T. Suda, and K. Oiwa, "Molecular communication: modeling noise effects on information rate," IEEE Transactions on NanoBioscience, vol. 8, no. 2, pp. 169-180, 2009.

[6] A. W. Eckford, "Timing information rates for active transport molecular communication," in Nano-Net, vol. 20 of Lecture Notes of the Institute for Computer Sciences, Social Informatics and Telecommunications Engineering, pp. 24-28, Springer Berlin Heidelberg, 2009.

[7] M. Pierobon and I. F. Akyildiz, "A physical end-to-end model for molecular communication in nanonetworks," IEEE Journal on Selected Areas in Communications, vol. 28, no. 4, pp. 602-611, 2010.

[8] A. W. Eckford, N. Farsad, S. Hiyama, and Y. Moritani, "Microchannel molecular communication with nanoscale carriers: Brownian motion versus active transport," in Proc. of the IEEE International Conference on Nanotechnology, (Seoul, South Korea), p. 5, 2010.

[9] N. Farsad, A. W. Eckford, S. Hiyama, and Y. Moritani, "Information rates of active propagation in microchannel molecular communication," in Proc. of the 5th International ICST Conference on Bio-Inspired Models of Network, Information, and Computing Systems, (Boston, MA), p. 7, 2010.

[10] T. M. Cover and J. A. Thomas, Elements of Information Theory 2nd Edition. Wiley-Interscience, 2 ed., 2006.

[11] R. Blahut, "Computation of channel capacity and rate-distortion functions," IEEE Transactions on Information Theory, vol. 18, no. 4, pp. 460-473, 1972.

[12] S. Arimoto, "An algorithm for computing the capacity of arbitrary discrete memoryless channels," IEEE Transactions on Information Theory, vol. 18 , no. 1 , pp. 14-20, 1972.

[13] S. X. Chen and J. S. Liu, "Statistical applications of the poissonbinomial and conditional bernoulli distributions," Statistica Sinica vol. 7, pp. 875-892, 1997.

[14] MATLAB, version 7.10.0 (R2010a). Natick, Massachusetts: The MathWorks Inc., 2010 